INTERNATIONAL JOURNAL
OHARMACEUTICAL SCIENCES
PESEARCH
RESER

Received on 10 June 2018; received in revised form, 14 February 2020; accepted, 23 February 2020; published 01 March 2020

\title{
ASSESSMENT OF FIXED DOSE COMBINATIONS IN A TERTIARY CARE TEACHING HOSPITAL
}

\author{
M. Kumaraswamy, R. Venkataraman, A. Samuel and J. C. Cherian *
}

Department of Pharmacy Practice, Sri Adichunchanagiri College of Pharmacy, Adichunchanagiri University, B. G. Nagara, Nagamangala Taluk, Mandya - 571448, Karnataka, India.

\section{Keywords:}

Fixed-Dose Combinations (FDCs), Central Drug Standard Control

Organization (CDSCO), World Health Organization (WHO), Active

Pharmaceutical Ingredients (APIs),

Essential Medicines List (EML),

National List of Essential Medicines (NLEM)

\section{Correspondence to Author: \\ Dr. Jubin Christy Cherian}

Doctor of Pharmacy (Pharm.D) Department of Pharmacy Practice, SAC College of Pharmacy,

B. G. Nagara, Nagamangala Taluk, Mandya - 571448, Karnataka, India.

E-mail: jubincherian1@gmail.com

\begin{abstract}
Fixed-dose combinations are defined as a combination of two or more active ingredients in a fixed ratio of doses. Recently fixed-dose combinations are more rapidly marketed; the available combinations in the market include both rational and irrational. The use of irrational fixed-dose combinations has resulted in severe adverse drug reactions and a reduction in patient quality of life. The main objective of this study was to assess the prescribing patterns and rationality of fixed-dose combinations in a tertiary care teaching hospital. A prospective and observational study was conducted in 249 inpatients after taking written consent from those who meet study criteria in the Medicine and Pediatric department for a period of 6 months. In this study, the combinations of drugs were equally distributed to both females and males patients (50\%), most patients were in the age group of 70-79 (20\%). A total of 355 combinations of drugs were used belonging to 48 different combinations. The most commonly prescribed combinations were Aspirin + Atorvastatin (13\%) and Tramadol + Acetaminophen (12\%). Out of which, 3 drug combinations were found to be banned accordingly to CDSCO Guidelines and WHO essential list of medicines. The rationality was assessed using 7 point criteria with a maximum scoring of 14.7 FDCs scored 0-8, 10 scored 9 to 11 and 31 scored 12 to 14 . This result indicated that the majority of fixed-dose combinations used were rational.
\end{abstract}

\section{INTRODUCTION: Fixed-Dose Combination} (FDC) is defined as a combination of two or more active ingredients in a fixed ratio of doses ${ }^{1}$. According to Food and Drug Administration USA defines a fixed-dose combination product as a product composed of any combination of drug and device or a biological product or a drug, device and biological product used for treatment ${ }^{2}$. With FDCs, drugs with complementary mechanisms of action from the different pharmacological groups should be aggregated ${ }^{3}$.

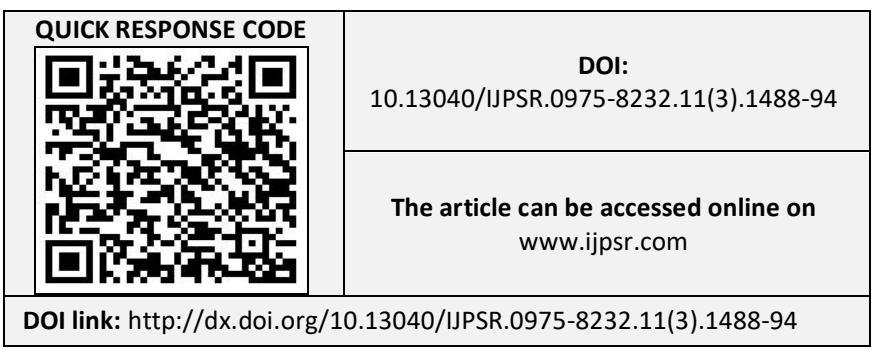

It must be safe and efficacious for the claimed indication ${ }^{3}$. Fixed-dose combinations are used worldwide and prescribed because it reduces pill burden, improves compliance, and it is costefficient ${ }^{2}$. Nowadays, irrational fixed-dose combinations are more rapidly being prescribed, which results in a serious adverse reaction and a reduction of the patient's quality of life increases cost and prolongs illness ${ }^{6}$.

The rationality of FDCs can be examined according to WHO guidelines; the FDCs were termed as rational if they had APIs with complementary mechanisms of a natural process, decrease antimicrobial resistance, increase efficacy and decrease the occurrence of adverse response and decrease the total cost of therapy ${ }^{6}$. Different studies conducted in India prove that many hospitals prescribing unapproved FDCs ${ }^{7}$. 
As per the Drug and Cosmetics Act and rule CDSCO is responsible for granting approval for Fixed-Dose Combination in India 5, 9. In March 2016, 344 FDCs were categorized as irrational and banned ${ }^{6}$. According to the national list of essential medicines in 2015, it contains $19 \mathrm{FDC}^{4}$. In order to promote rational use of medicines, the World health organization has implemented a concept of an essential drug list for the first time in 1977 and its regular updates of the model essential drug list for every two years ${ }^{8}$. Ishrar MG, Rathinavelu M and Reddy PY conducted an Interventional prospective study on FDC in anantapurau district at 27 community pharmacies over a period of 6 months. All information regarding demographic details was compiled in a suitably planned data collection form, first visit and second visit were conducted using ten-point questionnaires. They collected a 404 Fixed-Dose Combination and subjected to the evaluation of rationality using the standard data collection phase. Out of 404 FDCs, 144 meet the criteria of rationality, out of which just 35\% FDC's, which are rational, among them, $15 \%$ were outside the list of FDC's in the WHO Essential Medicine List and National List of Essential Medicines (NLEM). 70.4\% of FDCs are known to cause interactions between the constituent in a single FDC product. $93.9 \%$ drug is on the WHO list ${ }^{10}$. The seventeenth WHO model list of essential medicines contains only 25 approved FDCs ${ }^{11}$. FDCs are introduced every year in a large amount, and so knowledgeable about prescribing FDCs is important in order to provide better patient care ${ }^{11}$. Tertiary care teaching hospitals have a dual role to act in terms of educating medical staff and providing health maintenance facilities to patient ${ }^{12}$. Keeping this in the view we conducted a rational assessment on FDCs in a tertiary care teaching hospital.

\section{Objectives:}

- To assess the prescribing trend of the different Fixed-Dose Combinations.

- To evaluate the rationality of FDCs in the patients.

\section{MATERIALS AND METHODS:}

Study Site: Medicine and pediatrics department of a 1050 bedded Adichunchanagiri Hospital and Research Centre, B. G. Nagara.
Study Design: Prospective-observational study.

Study Period: Period of 6 months (September 2016 to March 2017).

Inclusion Criteria: All in patients who are admitted to medicine and pediatrics wards and receiving FDCs.

Exclusion Criteria: Patients not willing to participate and Out-patients on FDCs.

Data Collection: Data was collected after taking informed written consent from the patient or patient caretaker. The patient's medical records were reviewed from all units of Medicine, Pediatrics departments. The gathered data were entered in suitably designed data collection forms, which include demographic detail of the patients, past medical history including medications, final diagnosis, prescribed medicines, clinical laboratory data and present duration of therapy and route of administration, rationality assessment of FDC prescribed.

Data Analysis: The prescribing pattern was examined from the medication chart by analyzing a number of FDCs prescribed, FDC distribution among patients, FDCs prescribed in different disease conditions, the rationality of combination prescribed was valued by comparing with the list of FDCs approved by CDSCO and WHO guidelines and using Panda et al criteria. The dose of the individual Active Pharmaceutical Ingredients (APIs) was verified from standard textbooks and references in pharmacology and therapeutics.

Study Procedure: A comprehensive seven-point criterion developed by Panda et al. was used for the evaluation of the rationality of the FDCs. These criteria include all the dimensions of defining a rational FDC, and appropriate weighting (score) has been bound to each criterion. The total score thus obtained by an FDC will reflect its standing on the scale. The scoring criteria used in the study could award a maximum of 14 points to an FDC which indicates better rationality, and as the score reduces, the rationality to reduces. Each question carries a score of 2 .

1. The first point is that each API of the combination should preferably be in the Essential 
Medicines List (EML) of the WHO or in the National List of Essential Medicines (NLEM) of India.

2. The dose of each API should meet the requirements for a defined population group. The dose and the proportion of each API present in FDC should be appropriate for the intended use.

3. The combination should have the advantage of established evidence of efficacy and safety.

4. The overall cost of the combination should preferably be less than the cost of the individual components.

5. The FDC should facilitate either the reduction of the dose of individual drugs or their adverse effects.
6. The pharmacokinetic (PK) parameters of each API should not be affected. There should be no unfavorable PK interaction between the APIs. In the case of the PK parameters were different, the clinical benefits should be taken into consideration.

7. Finally, individual drugs should have different mechanisms of action.

RESULTS AND DISCUSSION: A total of 249 in patients with drug combinations were identified during the study period, out of which 125 (50\%) were males and $124(50 \%)$ females, giving a ratio of $1: 1$. The majority of the subjects came under the age group of 70-90, i.e., 62 subjects (25\%) in those 35 males and 27 females are there. The details are given in Table 1.

TABLE 1: DEMOGRAPHICS DETAILS OF THE STUDY POPULATIONS

\begin{tabular}{ccccc}
\hline Age Groups & Male & Female & Frequency(N) & Percentage (\%) \\
\hline $1-29$ & 12 & 13 & 25 & $10 \%$ \\
$30-39$ & 9 & 16 & 25 & $10 \%$ \\
$40-49$ & 26 & 21 & 47 & $19 \%$ \\
$50-59$ & 21 & 26 & 47 & $19 \%$ \\
$60-69$ & 22 & 21 & 43 & $17 \%$ \\
$70-90$ & 35 & 27 & 62 & $25 \%$ \\
Total & 125 & 124 & 249 & $100 \%$ \\
\hline
\end{tabular}

TABLE 2: FIXED DOSE COMBINATION OF DRUGS USED IN PATIENTS

\begin{tabular}{cccc}
\hline S. no. & Drug Combinations & No of prescriptions & Percent \\
\hline 1 & Atorvastatin + Aspirin & 47 & $13 \%$ \\
2 & Tramadol + Acetaminophen & 41 & $12 \%$ \\
3 & Pantoprazole + Domperidone & 31 & $9 \%$ \\
4 & Metformin + Glimepiride & 27 & $8 \%$ \\
5 & Telmisartan + Hydrochlorothiazide & 27 & $8 \%$ \\
6 & Spironolactone + Furosemide & 22 & $6 \%$ \\
7 & Theophylline + Etophylline & 20 & $5 \%$ \\
8 & Amoxicillin + Clavulanic acid & 16 & $4 \%$ \\
9 & Elemental iron + Folic acid & 12 & $3 \%$ \\
10 & Losartan + Hydrochlorothiazide & 10 & $3 \%$ \\
11 & Artesunate + Pyrimethamine + Sulfadoxine & 10 & $2 \%$ \\
12 & Ethambutol + Isoniazid + Pyrazinamide + Rifampicin & 9 & $1 \%$ \\
13 & Dicyclomine + Paracetamol & 7 & $1 \%$ \\
14 & Trypsin-chymotrypsin + Diclofenac & 6 & $1 \%$ \\
15 & Taurine + N-acetylcysteine & 6 & $1 \%$ \\
16 & Amlodipine + Atenolol & 5 & $1 \%$ \\
17 & Torsemide + Spironolactone & 5 & $1 \%$ \\
18 & Metformin + Pioglitazone & 4 & $1 \%$ \\
19 & Montelukast + Levocetirizine & 4 & $0.5 \%$ \\
20 & Aceclofenac + Thiocolchicoside & 4 & $0.5 \%$ \\
21 & Paracetamol + Aceclofenac & 3 & $0.5 \%$ \\
23 & Diclofenac + Serratiopeptidase & 3 & $0.5 \%$ \\
24 & Ranitidine + Domperidone & 3 & $0.5 \%$ \\
25 & Trimethoprim + Sulfamethoxazole & 3 & $0.5 \%$ \\
\hline
\end{tabular}




\begin{tabular}{lccc}
\hline 27 & Amlodipine + Hydrochlorothiazide & 2 & $0.5 \%$ \\
28 & Glipizide + Metformin & 2 & $0.5 \%$ \\
29 & Atenolol + Hydrochlorothiazide & 2 & $0.5 \%$ \\
30 & Metformin + Glibenclamide & 2 & $0.5 \%$ \\
31 & Other combinations & 18 & $5 \%$ \\
\hline
\end{tabular}

48 different combinations of FDC are used and some FDCs can be seen in more than one patient, so the total number of FDCs used in this study is 355. Among 355 combinations of drugs used, the combination of Atorvastatin and Aspirin is about $47(13 \%)$ prescribed for patients having cardiovascular disease, followed by tramadol and acetaminophen 41 (12\%) prescriptions done and also followed by other drugs as shown in Table 2.

Upon analyzing the distribution of FDCs, it has been found that out of 249 patients, 180 (73\%) patient received FDC with two active pharmaceutical ingredients, followed by $56(23 \%)$ patients received FDC with three active pharmacological ingredients whereas only 11 (4\%) patients received FDC with four active pharmacological ingredients. The details are shown in Fig. 1.

\section{FDC Distribution}

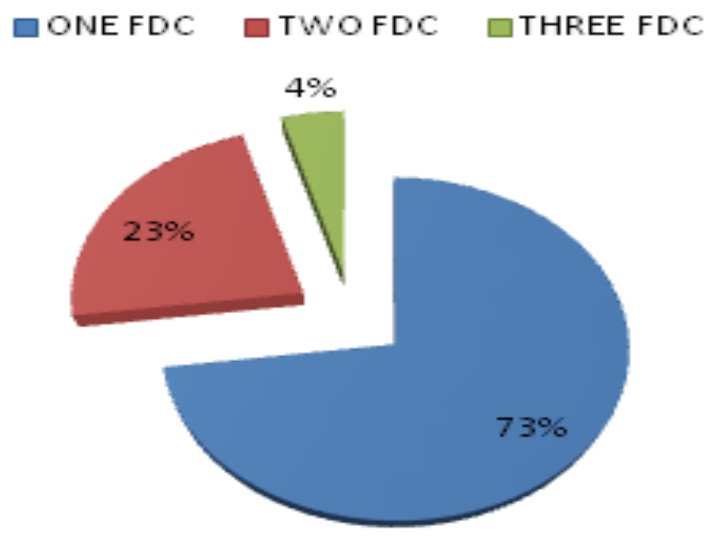

FIG. 1: FDC DISTRIBUTION

Out of 355 combinations, maximum combination $77(22 \%)$ were from cardiovascular disease followed by 68 (19\%), 43(12\%), 38(11\%), 30(8\%), $24(7 \%), 23(6 \%), 16(4 \%), 13(4 \%), 9(3 \%), 5(1 \%)$ combination from lower respiratory tract infections, viral fever, hypertension and diabetes mellitus, hypertension and lower respiratory infection, hematologic disorders, endocrine disorders, others, liver disease, central nervous system, kidney diseases, and gastrointestinal diseases and shown in Table 3.
TABLE 3: FDC DRUG PRESCRIBED IN DIFFERENT DISEASE CONDITIONS

\begin{tabular}{cccc}
\hline S. & Conditions & $\begin{array}{c}\text { No of FDC } \\
\text { Given }\end{array}$ & Percent \\
\hline 1 & Cardiovascular Disease & 77 & $22 \%$ \\
2 & Lower Respiratory Tract & 68 & $19 \%$ \\
& Infections & & \\
3 & Viral Fever & 43 & $12 \%$ \\
4 & Hypertension And & 38 & $11 \%$ \\
& Diabetes Mellitus & & \\
5 & Hypertension And Lower & 30 & $8 \%$ \\
& Respiratory Tract & & \\
\multicolumn{4}{c}{ Infection } \\
7 & Hematologic Disorders & 24 & $7 \%$ \\
8 & Endocrine Disorders & 23 & $6 \%$ \\
9 & Others & 16 & $4 \%$ \\
10 & Liver Disease & 13 & $4 \%$ \\
11 & Central Nervous System & 9 & $3 \%$ \\
12 & Kidney Diseases & 9 & $3 \%$ \\
\hline
\end{tabular}

In our study 48 different combinations used in this study, among which 3 drug combinations are found to be banned accordingly to CDSCO Guidelines and WHO essential list of medicines. 48 different combinations are used in this study and. These 3 combinations are Paracetamol and Aceclofenac, which is prescribed in three patients out of 249 followed by Metformin and Pioglitazone in four patients and a combination of Metformin + Pioglitazone + Glimepiride in one patient which are banned. Out of 48 combinations, 29 were approved accordingly by CDSCO, and WHO guidelines and 16 were not on the list, and three found to be banned. The details are shown in Tables $\mathbf{4}$ and $\mathbf{5}$.

\begin{tabular}{ccc} 
TABLE 4: RATIONALITY ASSESSMENT & \\
\hline FDC Prescribed & Frequency & Percent \\
\hline Rational FDC & 45 & $94 \%$ \\
Irrational FDC & 3 & $6 \%$ \\
Total & 48 & $100 \%$ \\
\hline
\end{tabular}

\begin{tabular}{ccc} 
TABLE 5: IRRATIONAL FDCS & \\
\hline $\begin{array}{c}\text { S. } \\
\text { no. }\end{array}$ & $\begin{array}{c}\text { Irrational Fixed } \\
\text { Dose Combinations }\end{array}$ & $\begin{array}{c}\text { No of FDC } \\
\text { Given }\end{array}$ \\
\hline 1 & Paracetamol + Aceclofenac & 3 \\
2 & Metformin + Pioglitazone & 4 \\
3 & Metformin + Pioglitazone + & 1 \\
& Glimepiride & \\
\hline
\end{tabular}


A comprehensive seven-point criterion developed by Panda et al., was used for the evaluation of the rationality of the FDCs. These criteria include all the dimensions of defining a rational FDC and appropriate weighting (score) has been attached to each criterion. The total score thus obtained by an FDC will reflect its standing on the scale. Seven FDCs scored 0-8, 10 FDCs scored 9 to 11 and 31 FDCs scored 12 to 14 . The details are shown in Table 6 and Fig. 2.

TABLE 6: SCORING BY PANDA ET AL CRITERIA FOR ASSESSING RATIONALITY OF FDC

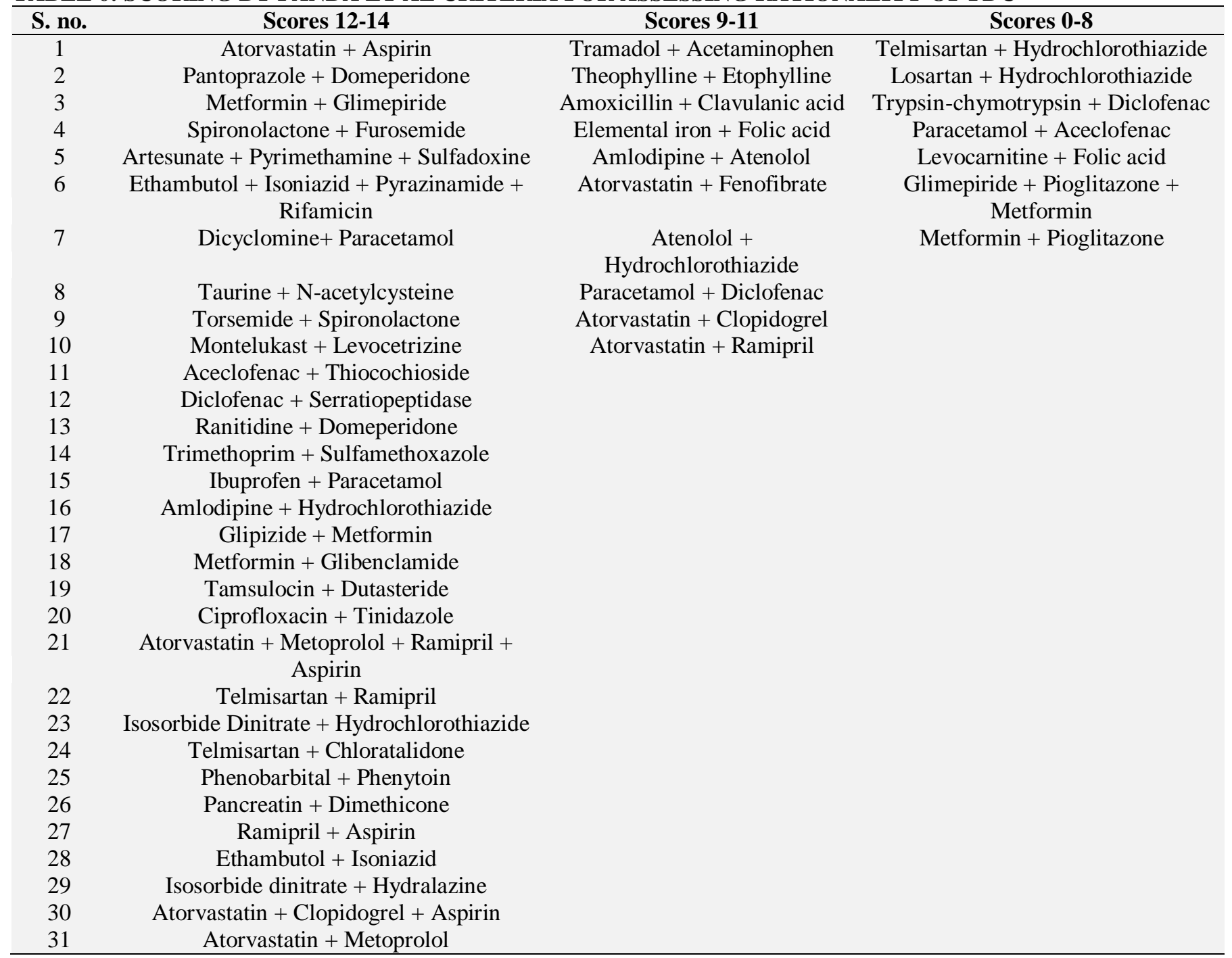

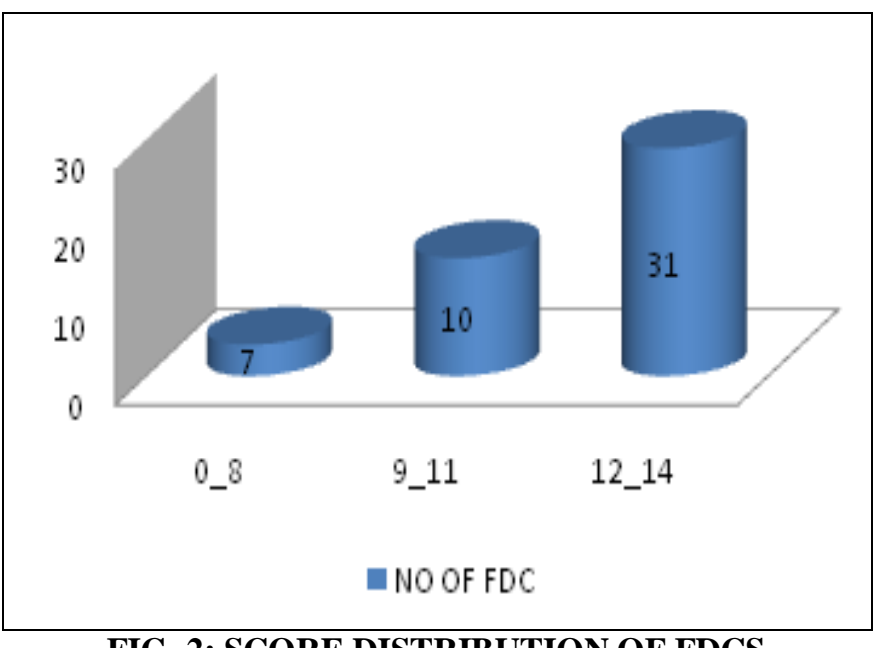

FIG. 2: SCORE DISTRIBUTION OF FDCS
DISCUSSION: The present study was conducted in the Medicine and Pediatrics Department of a tertiary care teaching hospital and assessed individual patients on FDCs. FDCs have many proven advantages over the use of monotherapy like improved efficacy, reduction in dose of individual drugs, reduced adverse drug reactions and improved patient compliance 13,14 . In our study, the male and female patients' distribution was more or less of equal proportions $(50 \%)$. The other studies conducted showed similar results ${ }^{15}$. The most common age group in the study was in 70-90 years age group which is in accordance with findings of other studies ${ }^{16}$. FDCs were most 
usually prescribed in age group $>50$ years, and this may be ascribed to the fact that this age is associated with numerous co-morbidities and disease progression and are poor compliance with their drug therapy. Among 249 patients, 48 different combinations of FDC are used and some received more than 1 FDC. The entire number of FDCs used in this study is $355.72 \%$ patients received 1 combination drugs, followed by $23 \%$ in 2 combination drugs and $4 \%$ in 3 combination drugs The most common FDC prescribed in the present study was Atorvastatin + Aspirin prescribed for patients experiencing cardiovascular disease. Maximum combination 77 (22\%) were from cardiovascular disease followed by 68 (19\%), 43 (12\%), 38 (11\%), 30 (8\%), 24 (7\%), 23 (6\%), 16 (4\%), $13(4 \%), 9(3 \%), 5(1 \%)$ combination from lower respiratory tract infections, viral fever, hypertension and diabetes mellitus, hypertension and lower respiratory infection, hematologic disorders, endocrine disorders, others, liver disease, central nervous system, kidney diseases, and gastrointestinal diseases. There were 333 (94\%) FDCs that had two API which was ground to be maximized, whereas only $12(3 \%)$ of combinations having three active pharmaceutical ingredients (API) whereas only 10 (3\%) combinations having 4 API, which is in accordance with determinations of other studies ${ }^{9}$.

In our study, analyzing the rationality of FixedDose Combination is an essential part, as we came across prescribing irrational or banned drugs, so it has to be assessed with the guidelines given by CDSCO and WHO essential list of medicines. In our study 48 different combinations used in this study, some FDCs are reported in more than one patient, so the total number of FDCs used in this study is 355 . Among these 3 drug combinations are found to be banned accordingly to CDSCO Guidelines and WHO essential list of medicines. 48 different combinations are used in this study and. These 3 combinations are Paracetamol and Aceclofenac, which was prescribed in 3 patients out of 249 followed by Metformin and Pioglitazone in 4 patients and a combination of Metformin + Pioglitazone + Glimepiride in one patient which are banned. Pioglitazone increases the risk of bladder cancer, heart failure, and eye problems and the risk of severe liver damage when paracetamol is used alone or in combination with others.
Out of 48 combinations, 29 were approved accordingly by CDSCO, and WHO guidelines and 16 were not on the list, and three found to be banned.

A comprehensive seven-point criterion developed by Panda et al., was used for the evaluation of the rationality of the FDCs. These criteria include all the dimensions of defining a rational FDC, and appropriate weighting (score) has been bound to each criterion. The total score thus obtained by an FDC will reflect its standing on the scale. The maximum score on the seven-point criteria for assessing the rationality of FDCs was 14, with each criterion carrying a score of 2, which is in accordance with findings of other studies 17.7 FDCs scored 0-8, 10 FDCs scored 9 to 11 , and 31 FDCs scored 12 to 14 .

CONCLUSION: On the basis of the comprehensive criteria, the study has made a systematic point-by-point evaluation of fixed-dose combinations. The fixed-dose combination is more rapidly being prescribed worldwide, the rationality of those combinations is an important factor to be considered. It was found that irrational FDCs may cause a serious adverse reaction or reduce the patient's quality of life. FDCs were mainly given to reduce pill burden and improve adherence of medicaments, but irrational combinations possess a high risk of adverse drug reaction it should carefully prescribe. There is always a continued need for prescribing patterns analysis in order to be updated about new trends in prescribing FDCs as well to provide better patient care.

ACKNOWLEDGEMENT: We would like to thank for the guidance and support received from all the members who contributed to this project, it was vital for the success of the project. We are grateful for their constant support and help.

We would like to extend our special thanks to HOD's of Medicine \& Pediatric of Adichunchanagiri Hospital and Research Centre, B.G. Nagara for their constant support. We would like to acknowledge the principal, staff members of Pharmacy practice department, SAC College of pharmacy, for providing necessary facilities to carry out this work.

CONFLICTS OF INTEREST: Nil 


\section{REFERENCES:}

1. Balat DJ, Gandhi MA, Patel P and Dikshit KR: Study of use fixed dose combinations in Ahmedabad, India. Indian Journal of Pharmacology 2014; 46: 503-09.

2. Gautam CS and Sahal: Fixed Dose Combinations; Rational or irrational: a view point. British Journal of Clinical Pharmacy 2008; 65: 795-96.

3. Banode S, Mehta M and Chourishi A: Rationality of FDC in Indian drug market: an evaluation at level of drug competition. Int J of Med Pharmal Science 2015; 9: 8-11.

4. Chandes S, Joshi A, Sonawani R and Jain N: A brief review on rational consideration of fixed-dose combination. Int Journal of Bio Research 2014; 6: 307-9.

5. Ghosh A, Malakar SK, Das S and Jha R: Assessment of use of FDC in indoor patients of medicine department of a tertiary care hospital in India. Global Journal for Research Analysis 2016; 5: 1-3.

6. Pisal SA, Jadhav S, Khanwelkar CC, Thorat MV and Matule MS: Study of fixed dose combinations in a tertiary care teaching hospital. Asian Journal of Pharmacology and Clinical Research 2016; 9: 35-6.

7. Sanz G, Fuster V, Guzman L, Guglietta A, Amaz AJ, Martinez F, Sarria A, Roncaglioni CM and Taubert K: Focus (Fixed Dose Combination drugs for secondary cardiovascular prevention) Report Summary. American Heart Journal 2011; 162: 811-17.

8. Upadhay D, Singh A, Joshi HS, Agarwal M and Katyal R: Study of prescriptions patterns of FDCs by medical internals. Int Journal of Bio Research. 2016; 7: 624-28.

9. Dalal K, Ganguly B and Gor A: Assessment of rationality of fixed dose combination approved in CDSCO list. Journal of Cli and Diagnostic Res 2016; 10: FC05-08.

10. Ishrar MG, Rathinvelu M and Reddy PY: Assessment of rational use of fixed-dose combination in community pharmacies of resources limited settings of Andhra
Pradesh. International Journal of Pharmaceutical Science and Research 2016; 440: 2181-86.

11. Aswini M, Mohanty BK, Hasamnis AA, Patil SS, Murty KSN and Jena SK: Prescription pattern in the department of medicine of a tertiary care hospital in South India. Journal of Clinical and Diagnostic Research 2010; 4: 2047-51.

12. Khjauria V, Tandon RV, Rani N, Roshi, Gupta S, Choudhary S and Gillani Z: Profile of adverse drug reactions with fixed dose combination: how big is the problem. Journal of Medical Education and Research 2015; 17: 33-7.

13. Bangalore S, Kamalakannan G, Parkar S and Messerli FH: Fixed dose combinations improve medication compliance: meta-analysis. Am Journal of Medi 2007; 120: 713-9.

14. Pan F, Chernew ME and Fendrick AM: Impact of fixeddose combination drugs on adherence to prescription medications. J Gen Intern Med 2008; 23: 611-614.

15. Pradhan S, Panda A, Sahu S and Ehera JP: An evaluation of prevalence and prescribing patterns of rational and irrational FDCs hospital based study. International Journal of Medical Science and Public Health 2016; 6: 58-62.

16. Connor J, Ratter $\mathrm{N}$ and Rodgers A: Do fixed dose combination pills or unit of packaging improve adherence systemic review. Bulletin of World Health Organisation 2001; 82: 935-39.

17. Balasubramaniam R, Hariharan D, Pamulapati VT, Devarajan V, Shanmugam S and Nai SAM: A Study on Evaluation of Rationality of Fixed Dose Combinations. American Journal of Pharmtech Research 2013; 3: 538-47.

18. Dakappa A and Narayanareddy M: A cross-sectional study to assess the rationality of Fixed Dose Combinations prescribed in geriatric patients in a tertiary care hospital. International Journal of Basic and Clinical Pharmacology 2016; 5: 1441-47.

How to cite this article:

Kumaraswamy M, Venkataraman R, Samuel A and Cherian JC: Assessment of fixed dose combinations in a tertiary care teaching hospital. Int J Pharm Sci \& Res 2020; 11(3): 1488-94. doi: 10.13040/IJPSR.0975-8232.11(3).1488-94.

All $\odot 2013$ are reserved by the International Journal of Pharmaceutical Sciences and Research. This Journal licensed under a Creative Commons Attribution-NonCommercial-ShareAlike 3.0 Unported License

This article can be downloaded to Android OS based mobile. Scan QR Code using Code/Bar Scanner from your mobile. (Scanners are available on Google Playstore) 